\title{
Exponentials of Sums of Operators in a Banach Space
}

\author{
Michael Gil'
}

(Communicated by Salvador ROMAGUERA)

\begin{abstract}
Let $A$ and $B$ be bounded linear operators in a Banach space. We consider the following problem: if $\int_{0}^{\infty}\left\|e^{A t}\right\|\left\|e^{B t}\right\| d t<\infty$, under what conditions we have $\int_{0}^{\infty}\left\|e^{(A+B) t}\right\| d t<\infty$ ? Our main result is formulated in terms of the norm of the commutator $A B-B A$.

Keywords: Banach space, linear operators, exponential, stability.

AMS Subject Classification (2010): Primary: 47A10; Secondary: 47B10; $15 A 42$.

${ }^{*}$ Corresponding author
\end{abstract}

\section{Introduction and statement of the main result}

Let $\mathcal{X}$ be a Banach space with a norm $\|\cdot\|$ and $\mathcal{B}(\mathcal{X})$ the algebra of bounded linear operators in $\mathcal{X}$. Denote by $\sigma(A)$ the spectrum of $A \in \mathcal{B}(\mathcal{X})$.

We consider the following problem: let $A, B \in \mathcal{B}(\mathcal{X})$ and

$$
J:=\int_{0}^{\infty}\left\|e^{A t}\right\|\left\|e^{B t}\right\| d t<\infty .
$$

What conditions provide the inequality $\int_{0}^{\infty}\left\|e^{(A+B) t}\right\| d t<\infty$ ? Since the considered operators are bounded, the problem can be reformulated in the following way: if $\alpha(A):=\sup \Re \sigma(A)<0$, under what conditions we have $\alpha(A+B)<0$ ?

The theory of the spectrum of sums of operators has a long story, cf. [1, 8-11,13] and references given therein, but mainly sums of selfadjoint operators have been investigated. The sums of pseudo-hermitian matrices were considered in [3]. The paper [12] is devoted to sums of the eigenvalues of random matrices. In the paper [6], non-selfadjoint operators in a Hilbert space are considered. The approach presented in this paper is absolutely different from the approach of [6].

As it is well-known, the inequality $\alpha(A+B)<0$ provides the stability conditions of the differential equation

$$
\frac{d w(t)}{d t}=(A+B) w(t) \quad(t \geq 0)
$$

Various integro-differential equations are examples of such equations, cf. [5]. To the best of our knowledge in the available literature that equation is investigated as a perturbation the equation

$$
\frac{d u(t)}{d t}=A u(t) \quad(t \geq 0)
$$

cf. [2] and references given therein. Besides the commutator $K=A B-B A$ does not play any role. At the same time our stability conditions are formulated in terms of the norm of the commutator. As it is shown below, in the appropriate situations this fact allows us to improve stability conditions obtained by the traditional perturbations.

Received : 10-April-2017, Accepted : 21-July-2017 
Put $X(t)=e^{(A+B) t}, Y(t)=e^{A t} e^{B t}(t \geq 0)$,

$$
\|X\|_{C}:=\sup _{s \geq 0}\|X(s)\| \text { and }\|X\|_{L^{1}}:=\int_{0}^{\infty}\|X(s)\| d s .
$$

Clearly, $\|Y\|_{C}<\infty$ and $\|Y\|_{L^{1}}<J$, provided condition (1.1) holds. Now we are in a position to formulate our main result.

Theorem 1.1. Let the conditions (1.1) and

$$
\|K\| J^{2}<1
$$

hold. Then

$$
\|Y-X\|_{C} \leq \frac{\|K\| J^{2}\|Y\|_{C}}{1-\|K\| J^{2}}
$$

and

$$
\|Y-X\|_{L^{1}} \leq \frac{J^{3}\|K\|}{1-\|K\| J^{2}}
$$

This theorem is proved in the next section. It is sharp: if $K=0$, then $X(t)=Y(t)$. Since $\|Y\|_{L^{1}}<J$, from (1.3) and (1.4) it directly follows that

$$
\|X\|_{C} \leq \frac{\|Y\|_{C}}{1-\|K\| J^{2}}
$$

and

$$
\|X\|_{L^{1}} \leq \frac{J}{1-\|K\| J^{2}}
$$

For instance, let

$$
\left\|e^{A t}\right\| \leq c_{A} e^{\alpha_{A} t},\left\|e^{B t}\right\| \leq c_{B} e^{\alpha_{B} t}\left(c_{A}, c_{B}=\text { const } \geq 1 ; \alpha_{A}, \alpha_{B} \text { are real constants } ; t \geq 0\right),
$$

and $\alpha_{A}+\alpha_{B}<0$. Then

$$
J \leq \frac{c_{A} c_{B}}{\left|\alpha_{A}+\alpha_{B}\right|}
$$

Now we can directly apply Theorem 1.1.

Let us compare our results with the traditional perturbation method. To this end consider a simple example. Let $\mathcal{X}=C(0,1)$ be the space of continuous scalar functions defined on $[0,1]$ with the sup-norm, $A u(x)=a(x) u(x)$ and $B u(x)=b(x) u(x)(u(.) \in C(0,1))$, where $a(),. b(.) \in C(0,1)$.

Under consideration $\left\|e^{A t}\right\| \leq e^{\alpha(A) t},\left\|e^{B t}\right\| \leq e^{\alpha(B) t}(t \geq 0)$ with $\alpha(A):=\sup _{x} \Re a(x)$ and $\alpha(B):=\sup _{x} \Re b(x)$. Put $\tilde{A}=A+B$. By the traditional perturbation method, applying the equality

$$
e^{\tilde{A} t}-e^{A t}=\int_{0}^{t} e^{A(t-s)} B e^{\tilde{A} s} d s
$$

we obtain

$$
\begin{aligned}
& \left\|e^{\tilde{A} t}\right\| \leq\left\|e^{A t}\right\|+\int_{0}^{t}\left\|e^{A(t-s)}\right\|\|B\|\left\|e^{\tilde{A} s}\right\| d s \\
& \leq e^{\alpha(A) t}+e^{\alpha(A) t} \int_{0}^{t}\left\|e^{\tilde{A} s}\right\|\|B\| e^{-\alpha(A) s} d s .
\end{aligned}
$$

Simple calculations show that $\left\|e^{\tilde{A} t}\right\|=\left\|e^{(A+B) t}\right\| \leq e^{(\alpha(A)+\|B\|) t}$. Thus the perturbation method gives us the stability condition $\alpha(A)+\|B\|<0$. At the same time stability condition following from Theorem 1.1 is $\alpha(A)+\alpha(B)<0$. 


\section{Proof of Theorem 1.1}

Note that

$$
\frac{d X(t)}{d t}=(A+B) X(t)
$$

and

$$
\frac{d Y(t)}{d t}=A e^{A t} e^{B t}+e^{A t} B e^{B t}=(A+B) e^{A t} e^{B t}+e^{A t} B e^{B t}-B e^{A t} e^{B t}=(A+B) Y(t)+F(t),
$$

where $F(t)=\left[e^{A t}, B\right] e^{B t}$. Subtracting (2.1) from (2.2), we get

$$
\frac{d(Y(t)-X(t))}{d t}=(A+B)(Y(t)-X(t))+F(t) .
$$

Consequently,

$$
Y(t)-X(t)=\int_{0}^{t} e^{(A+B)(t-s)} F(s) d s=\int_{0}^{t} X(t-s) F(s) d s
$$

Hence,

$$
\|Y(t)-X(t)\| \leq \int_{0}^{t}\|X(t-s)\|\|F(s)\| d s
$$

and therefore

$$
\|X(t)\| \leq\|Y(t)\|+\int_{0}^{t}\|X(t-s)\|\|F(s)\| d s .
$$

Making use of (2.4), for any finite $t>0$ we can write

$$
\sup _{s \leq t}\|X(s)\| \leq \sup _{s \leq t}\|Y(s)\|+\sup _{s \leq t}\|X(s)\| \int_{0}^{t}\|F(s)\| d s .
$$

As is checked in [7],

$$
\left[e^{A t}, B\right]=\int_{0}^{t} e^{A(t-s)} K e^{A s} d s
$$

Hence,

$$
\|F(t)\| \leq\left\|\left[e^{A t}, B\right]\right\|\left\|e^{B t}\right\| \leq\left\|e^{B t}\right\| \int_{0}^{t}\left\|e^{A(t-s)}\right\|\|K\|\left\|e^{A s}\right\| d s .
$$

Then

$$
\begin{gathered}
\|F\|_{L^{1}} \leq\|K\| \int_{0}^{\infty}\left\|e^{B t}\right\| \int_{0}^{t}\left\|e^{A(t-s)}\right\|\left\|e^{A s}\right\| d s d t \\
=\|K\| \int_{0}^{\infty}\left\|e^{A s}\right\| \int_{s}^{\infty}\left\|e^{B t}\right\|\left\|e^{A(t-s)}\right\| d t d s \\
=\|K\| \int_{0}^{\infty}\left\|e^{A s}\right\| \int_{0}^{\infty}\left\|e^{B\left(t_{1}+s\right)}\right\|\left\|e^{A t_{1}}\right\| d s d t_{1} \\
\leq\|K\| \int_{0}^{\infty}\left\|e^{A s}\right\|\left\|e^{B s}\right\| d s \int_{0}^{\infty}\left\|e^{B t_{1}}\right\|\left\|e^{A t_{1}}\right\| d t_{1}=J^{2}\|K\| .
\end{gathered}
$$

So

$$
\|F\|_{L^{1}} \leq J^{2}\|K\|<\infty .
$$

Under condition (1.2), inequalities (2.5) and (2.6) imply (1.5). In addition, by (2.4)

$$
\begin{gathered}
\int_{0}^{t}\left\|X\left(t_{1}\right)\right\| d t_{1} \leq \int_{0}^{t}\left\|Y\left(t_{1}\right)\right\| d t_{1}+\int_{0}^{t} \int_{0}^{t_{1}}\left\|X\left(t_{1}-s\right)\right\|\|F(s)\| d s d t_{1} \\
\leq J+\int_{0}^{t}\|F(s)\| \int_{s}^{t}\left\|X\left(t_{1}-s\right)\right\| d t_{1} d s \leq J+\int_{0}^{\infty}\|F(s)\| d s \int_{0}^{t}\left\|X\left(t_{1}\right)\right\| d t_{1} .
\end{gathered}
$$


Hence according to (2.6) and (1.2) we arrive at (1.6). From (2.3) and (2.6) we obtain

$$
\|Y-X\|_{C} \leq\|X\|_{C} \int_{0}^{\infty}\|F(s)\| d s \leq\|X\|_{C} J^{2}\|K\|
$$

Now (1.5) implies (1.3). Moreover, due to (2.3) and (2.6),

$$
\begin{gathered}
\int_{0}^{\infty}\left\|X\left(t_{1}\right)-Y\left(t_{1}\right)\right\| d t_{1} \leq \int_{0}^{\infty} \int_{0}^{t_{1}}\|X(t-s)\|\|F(s)\| d s d t_{1} \leq \\
\int_{0}^{\infty}\|F(s)\| \int_{s}^{t}\|X(t-s)\| d t d s \leq \int_{0}^{\infty}\|F(s)\| d t \int_{0}^{\infty}\|X(t)\| d t \leq\|K\| J^{2}\|X\|_{L^{1}} .
\end{gathered}
$$

Now (1.6) implies (1.4), as claimed.

\section{Particular cases}

\section{The finite dimensional operators}

In this subsection $A$ and $B$ are $n \times n$ matrices. Put

$$
g(A)=\left[N_{2}^{2}(A)-\sum_{k=1}^{n}\left|\lambda_{k}(A)\right|^{2}\right]^{1 / 2},
$$

where $\lambda_{k}(A)(k=1, \ldots, n)$ are the eigenvalues of $A$, counted with their multiplicities; $N_{2}(A)=\left(\operatorname{trace} A A^{*}\right)^{1 / 2}$ is the Frobenius (Hilbert-Schmidt) norm of $A, A^{*}$ is the adjoint operator. The following relations are checked in [4, Section 2.1]: $g^{2}(A) \leq N_{2}^{2}(A)-\left|\operatorname{trace} A^{2}\right|$,

$$
g\left(e^{i \tau} A+z I\right)=g(A)(\tau \in \mathbb{R}, z \in \mathbb{C}) \text { and } g^{2}(A) \leq \frac{N_{2}^{2}\left(A-A^{*}\right)}{2} .
$$

If $A$ is a normal matrix, then $g(A)=0$. It is shown in [4, Example 2.7.3] that

$$
\left\|e^{A t}\right\| \leq e^{\alpha(A) t} \sum_{k=0}^{n-1} \frac{t^{k} g^{k}(A)}{(k !)^{3 / 2}}(t \geq 0) .
$$

Assume that $\alpha_{0}=\alpha(A)+\alpha(B)<0$. Then

$$
\|J\| \leq \int_{0}^{\infty} e^{\alpha_{0} t}\left(\sum_{j, k=0}^{n-1} \frac{t^{k+j} g^{j}(A) g^{k}(B)}{(k ! j !)^{3 / 2}}\right) d t .
$$

Thus $J \leq J_{0}$, where

$$
J_{0}:=\sum_{j, k=0}^{n-1} \frac{g^{j}(A) g^{k}(B)(k+j) !}{\left|\alpha_{0}\right|^{j+k+1}(j ! k !)^{3 / 2}} .
$$

Now we can directly apply Theorem 1.1. If $A$ is normal, then $g(A)=0$ and

$$
J_{0}=\sum_{k=0}^{n-1} \frac{g^{k}(B)}{\left|\alpha_{0}\right|^{k+1}(k !)^{1 / 2}} .
$$

Besides we take $0^{0}=1$. If both $A$ and $B$ are normal, then $J_{0}=\frac{1}{\left|\alpha_{0}\right|}$. 


\section{Infinite-dimensional operators}

In this subsection $\mathcal{X}$ is a separable Hilbert space. Suppose that $\Im A=\left(A-A^{*}\right) / 2 i$ and $\Im B$ are Hilbert-Schmidt operators, i.e. $N_{2}(\Im A)=\left(\operatorname{trace}(\Im A)^{2}\right)^{1 / 2}<\infty$. As is shown in [4, Example 7.10.3],

$$
\left\|e^{A t}\right\| \leq e^{\alpha(A) t} \sum_{m=0}^{\infty} \frac{t^{m} u^{m}(A)}{(m !)^{3 / 2}}(t \geq 0),
$$

where $u(A)=\sqrt{2} N_{2}(\Im A)$. Again assume that $\alpha_{0}=\alpha(A)+\alpha(B)<0$. Then $J \leq J_{2}$, where

$$
J_{2}:=\int_{0}^{\infty} e^{\alpha_{0} s} \sum_{j, k=0}^{\infty} \frac{s^{k+j} u^{j}(A) u^{k}(B)}{(j ! k !)^{3 / 2}} d s .
$$

The simple calculations show that

$$
J_{2}=\sum_{j, k=0}^{\infty} \frac{(k+j) ! u^{j}(A) u^{k}(B)}{\left|\alpha_{0}\right|^{k+j+1}(j ! k !)^{3 / 2}} .
$$

Now one can directly apply Theorem 1.1.

\section{References}

[1] Bercovici, H. and Li Wing Suet, Inequalities for eigenvalues of sums in a von Neumann algebra. In Recent Advances in Operator theory and Related topics (Szeged, 1999), pp. 113-126, Oper. Theory Adv. Appl., 127, Birkhauser, Basel, 2001.

[2] Daleckii, Yu L. and Krein, M.G. Stability of Solutions of Differential Equations in Banach Space, Amer. Math. Soc., Providence, R. I. 1974.

[3] Foth, P. Eigenvalues of sums of pseudo-hermitian matrices, Electronic Journal of Linear Algebra, 20 (2010) $115-125$.

[4] Gil', M.I. Operator Functions and Localization of Spectra, Lecture Notes In Mathematics vol. 1830, SpringerVerlag, Berlin, 2003.

[5] Gil', M.I. On stability of linear Barbashin type integro-differential equations, Mathematical Problems in Engineering, vol. 2015, Article ID 962565, (2015), 5 pages.

[6] Gil', M.I. Stability of sums of operators, Ann. Univ. Ferrara, 62, (2016) 61-70.

[7] Gil', M.I. A bound for the Hilbert-Schmidt norm of generalized commutators of nonself-adjoint operators, Operators and Matrices, 11, no. 1 (2017) , 115-123

[8] Helmke, U. and Rosenthal, J. Eigenvalue inequalities and Schubert calculus, Math. Nachr. 171 (1995), 207-225.

[9] Horn, A. Eigenvalues of sums of Hermitian matrices, Pacific J. Math. 12 (1962), 225-241.

[10] Lidskii, B.V. Spectral polyhedron of the sum of two Hermitian matrices, Funct. Anal. Appl. 16 (1982), 139-140.

[11] Miranda, H. Diagonals and eigenvalues of sums of Hermitian matrices. Extreme cases. Proyecciones 22, no. $2,(2003) 127-134$.

[12] Peacock, M.J.M., Collings I.B. and Honig, M.L. Eigenvalue distributions of sums and products of large random matrices via incremental matrix expansions, IEEE Trans. on Information Theory, 54, no. 5, (2008) 2123-2137

[13] Thompson, R.C. and Freede, L. On the eigenvalues of a sum of Hermitian matrices, Linear Algebra Appl. 4 (1971), 369-376. 


\section{Affiliations}

MiCHAEL GIL'

ADDRESS: Department of Mathematics, Ben Gurion University of the Negev, P.0. Box 653, Beer-Sheva 84105, Israel. E-MAIL: gilmi@bezeqint.net

ORCID ID: orcid.org/0000-0002-6404-9618 